\title{
REFLEXIONES PARA CONSTRUIR ARREGLOS INSTITUCIONALES DE RESPONSABILIDAD TURÍSTICA DIGITAL DESDE LA PERSPECTIVA DE LA TEORÍA DE LA ELECCIÓN RACIONAL Y LA ESCALA LOCAL
}

\author{
LUCÍA GONZÁLEZ TORREROS \\ lucia.gtorreros@academicos.udg.mx \\ José LUIS SANTANA MEDINA \\ jlsantana@cucea.udg.mx \\ Universidad de Guadalajara
}

Este trabajo busca reflexionar sobre la posibilidad de que los agentes sociales establezcan arreglos institucionales para delimitar la acción y la toma de decisiones carentes de sentido ético y favorecer así el desempeño de la tecnología en la era digital en el sector turístico. Bajo un enfoque cualitativo: empírico-descriptivo y teórico, con apoyo de la Teoría de Elección Racional, se propone una vía para el diseño y definición de la Responsabilidad Turística Digital, como una institución forjada desde la escala local.

Palabras clave: racionalidad, responsabilidad, arreglos, escala local, turismo.

\section{REFLECTIONS FOR BUILDING INSTITUTIONAL ARRANGEMENTS ABOUT TOURISTIC DIGITAL RESPONSIBILITY BASED ON THE RATIONAL ELECTION THEORY AND LOCAL SCALE}

This research seeks reflection on the possibility that social agents must set institutional fixes towards action framing and decision making lacking ethical sense, thus favoring technological development on the digital era in the touristic sector. Under a qualitative focus: empirical-descriptive and theoretical, with the support of the Rational Election Theory, a design and definition of the Digital Touristic Responsibility is proposed, as an institution forged at a local scale.

Keywords: rationality, responsibility, arrangements, local scale, tourism. 


\section{Introducción}

T odas las Revoluciones Industriales (RI) han implicado cambios socioterritoriales importantes que se imprimen en la evolución de la actividad turística. La primera RI tuvo como característica la invención de la máquina de vapor y la construcción del ferrocarril. La segunda, unos 100 años después, supuso un desarrollo impulsado por la electricidad y la cadena de montaje; entre esta y la tercera transcurrieron poco más de 60 años. La tercera, que se marca con la definición de la Inteligencia Artificial (IA), sucede en el año de 1956, mientras que la cuarta, calificada en el Foro Económico Mundial de 2016 apenas unas décadas después, significó la vertiginosa incorporación a la vida diaria de un conjunto de sistemas hiperconectados, principalmente por la World Wide Web (www), fenómeno que desdibujó fronteras físicas y redibujó las interacciones sociales y económicas del mundo real (Schwab, 2016).

En el campo de la evolución del turismo, las dos primeras contribuyeron a la democratización y al turismo de masas, en tanto que las dos últimas han marcado la oportunidad de incrementar la conexión en tiempo real, con la amplia posibilidad de generar experiencias personalizadas, pero también un uso encubierto de datos personales.

La tendencia actual se vincula con los esfuerzos y ambientes cognitivos y digitales de la IA, pero trae a la escena una renovada participación humana, particularmente en un sentido ético en las acciones y toma de decisiones, así como la necesidad de un nuevo contrato social.

De acuerdo con lo que algunos expertos sugieren, la humanidad se encuentra en la antesala de la Sociedad 5.0 como proyecto social, al argumentar que la ética nunca debió perderse de vista en las llamadas revoluciones tecnológicas, misma que había sido silenciada, abandonada (Arencibia et al., 2020). El reposicionamiento de la ética y las personas fundamenta un proyecto/estrategia que tuvo su impulso en Japón en 2015, al "colocar a los seres humanos en el centro de la producción industrial... [sic]..., permitirá a la industria 5.0 brindar productos que desean los consumidores y crear empleos más significativos..." (Østergaard, 2019, citado en Arencibia et al., 2020, p. 103).

Si bien es cierto que la influencia de la tecnología se cuenta con éxitos, este escenario deja al descubierto la falta de acuerdos que permitan conciliar el potencial 
que ofrece la tecnología con la protección de los usuarios y sus datos personales, lo que ha conducido a situaciones poco deseables. Esta cuestión se tornó en objetivo de reflexión de los líderes reunidos en la Cumbre de Davos de 2016, lo que motivó en la primera década del siglo XXI a pensar la responsabilidad digital (Schwab, 2016).

Para iniciar un camino argumentativo, se exponen brevemente algunas declaraciones particulares: Berners-Lee, creador de la Web en 1989, detalló que a pesar del optimismo por la web se identifica el abuso con los datos personales y un poder centralizado en las empresas de tecnología, apuntando hacia la necesidad de un nuevo contrato social fundamentado en la responsabilidad (WEForum, 2021).

Un ejemplo de esta preocupación se ofrece a partir de la nota publicada en el sitio Infolat (2021) en la cual se cuenta sobre la vulnerabilidad en el uso de la aplicación Google. Esta situación puso en riesgo a más de cinco millones de usuarios: hackeo robo de datos, acceso a correos, historial y realización de llamadas y de búsquedas e incluso la activación de cámaras.

En una mesa de expertos de INVAT-TUR (2021) en la cual se discutió sobre el papel de la IA en el sector turístico, se señaló entre otros asuntos para reflexionar el creciente interés por los datos de los turistas, con el ejemplo de la existencia de robots que deciden los precios según el ingreso económico, así como el monitoreo de la actividad en el destino, lo que supone una invasión a la privacidad.

Se insiste en que la IA no es neutra, trabaja para alguien más, quien por supuesto no es el turista. Otros argumentos presentados radican en que la tecnología manipula las decisiones respecto a lo que dicho turista hace en el destino, modula sus motivaciones y deseos de experiencias, la neurolingüística aplicada. Sus datos personales constituyen la mercancía más valiosa, la gratuidad de la aplicaciones y productos digitales no es para nada generosa, significa que el producto que se comercializa en realidad son las personas y sus datos.

En el documental El dilema de las redes sociales (Orlowski, 2020) se advierte sobre varios peligros, uno en particular apunta a la IA; esta crea realidades individuales que alejan al usuario de la realidad colectiva, lo distancia del mundo real y del sentido de comunidad. La realidad es entonces en extremo individual, la que muestra y reproduce la máquina. La información que el individuo recibe es enormemente 
selectiva y dirigida, lo que influye en su racionalidad, se manipula su estado de ánimo y se explota la vulnerabilidad, en este caso, de los turistas.

En un asunto paralelo, a principios del año 2021 en México se modifica la Ley de Telecomunicaciones y Radiodifusión, con la que se pretende recopilar 10 datos personales -entre los que se encuentran los biométricos- de los usuarios de telefonía celular (DOF, 2021). En este contexto, la Ley de Protección de Datos Personales en Posesión de Particulares (DOF, 2010) resulta insuficiente, al considerar de una forma ligera y hasta ingenua la posibilidad de delitos digitales.

Del sitio de DataTur (2015) se revisan los estudios de Big Data que forman parte de los Documentos de Investigación Estadística y Económica realizados en colaboración con BBVA-Bancomer y BBVA Data \& Analytics. A pesar de que son datos personales de clientes y usarios de redes sociales, la entidad propietaria de los datos es BBVA Data \& Analitycs, se señala que esto es en apego a la Ley de Protección de Datos. Los datos deberían ser propiedad de los usuarios de aplicaciones y no ser sujetos de apropiación por terceros. Como un caso específico más reciente, la agencia Reportur.mx (2019) ofrece un reportaje titulado: Masivo robo de datos en hoteles y agencias como Booking, evidenciando que la exposición y el riesgo se ha potenciado con la pandemia del COVID-19, la vulnerabilidad de los usuarios es cada vez mayor.

En este sentido, la tesis de trabajo que se propone es que las tecnologías se instalan de manera acelerada en la cotidianidad turística, con diversas repercusiones en las prácticas y territorios, de ahí la importancia de establecer arreglos institucionales vinculados con la Responsabilidad Turística Digital (en adelante RTD), en los que participen los agentes sociales en el ámbito local.

La hipótesis plantea que si el turismo es una práctica que se produce localmente, por ende esta escala se presenta como el ámbito ideal para la generación de sinergias entre los distintos agentes sociales del sector; si es así, debería ofrecer un ambiente de ética y responsabilidad para debatir y dar respuesta a las preocupaciones respecto a la dinámica de la tecnología en dicho contexto, para compartir información y reflexionar sobre las distintas racionalidades, una vía que les permita tomar las mejores decisiones de interés colectivo.

Lo anterior supone cuestionarse respecto de ¿cuáles son los contenidos de la RTD que deberán ser incorporados como parte de un arreglo institucional, para que 
cumplan con el cometido de guiar a los agentes que interactúan en la escala local, en los procesos de toma de decisiones? de forma que sea posible disminuir los riesgos que derivan del uso de estas tecnologías. Se plantea como objetivo: reflexionar a partir de la Teoría de Elección Racional (TER) y la racionalidad de los agentes sociales del turismo la posibilidad de llegar a arreglos institucionales y proponer algunas vías, desde la escala local, para el diseño y la definición de la RTD.

No es la intención de este trabajo realizar un debate teórico profundo sobre la TER, sino establecer las bases para comprender lo que debe ponerse sobre la mesa de diálogo, para dar paso a los arreglos institucionales y reconocer las distintas racionalidades que entran en juego del campo turístico; con esto se espera contribuir a robustecer la teorización del turismo y lograr una mejor comprensión de las distintas formas en que la tecnología y el turismo interactúan.

En este sentido, el presente documento se divide en cinco apartados: primeramente se presenta una breve introducción, seguida de la metodología; en tercer lugar se integra la revisión teórica de la elección racional (TER) acompañada de una argumentación sobre la escala local y la responsabilidad. En un cuarto punto se expone el contexto actual sobre la actividad turística, la reflexión sobre la contradicción existente entre la hiperconexión de los turistas y la exposición y uso de datos personales; en el mismo se visualizan algunas vías de acción para la definición de la RTD desde la escala local. Finalmente, se desarrollan las principales conclusiones.

\section{Metodología}

En esta investigación se utiliza básicamente una metodología de aproximación cualitativa con dos enfoques: empírico-descriptivo y teórico. El primero está fundamentado en una revisión y descripción de distintas evidencias, las cuales sugieren el impacto negativo del uso de la tecnología que realizan las personas en su rol de turistas, empresarios o gestores de los destinos turísticos. El segundo se presenta a partir de la lectura de textos académicos consultados siguiendo la línea de tres categorías teóricas: elección racional (Weber, 1928; Simon, 1955 y 2007; Elster, 2005; North, 1971 y 1994; Ostrom, 2015), escala local y responsabilidad social (Misseri, 2012 y Bowen, 1953, a través de Acquier et al., 2011) tal como se presenta en la figura 1. 
Figura 1. Categorías y variables de la investigación

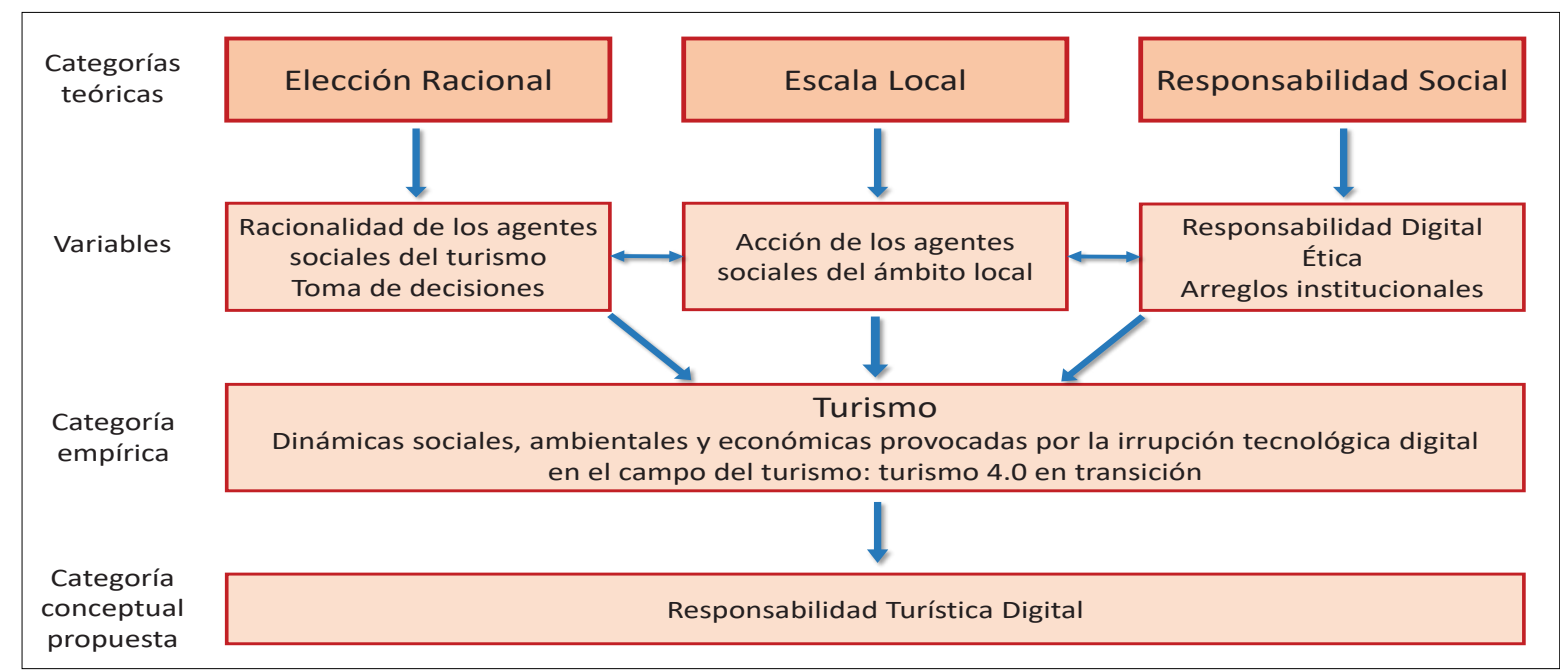

Fuente: Elaboración propia.

\section{Los pilares teóricos de la RTD}

\subsection{La Teoría de Elección Racional}

Los individuos poseen libertad de acción, son críticos y cuentan con iniciativa, tienen capacidad cognitiva, aportan innovación y son capaces de inducir cambios tanto en la cultura como en las estructuras socio-territoriales; al mismo tiempo, actúan bajo principios y racionalidades que condicionan su toma de decisiones. Estas dinámicas de interrelación simbiótica manifiestan una esencia compleja de la realidad. La TER forma parte del debate científico, nutriéndose de las aportaciones de destacados teóricos como Max Weber, Herbert A. Simon, Jon Elster, Douglas North y Elinor Ostrom.

Entre las contribuciones más tempranas se encuentra la de Max Weber (1928), su reflexión resulta de analizar el papel de la ética de las religiones en el origen del capitalismo. Subraya que el hombre es solo un administrador de lo que ha recibido de Dios y, por lo tanto, su cuidado se justifica y responde al valor depositado acorde con los principios de la racionalidad capitalista.

En este contexto, el summun bonum (fin supremo) de esta ética era ganar más y más dinero. Por ello, esta perspectiva se fundamenta en la acción individual del hombre y se guía por la maximización económica. Acorde con Aguilar (2020), aun- 
que la acción racional weberiana es individual, también puede ser colectiva y social, siendo intencional en todos los casos. Lo anterior implica una acción causal, es decir, conlleva el cálculo de los efectos de sus acciones, la búsqueda de ganancias o beneficios.

Herbert Simon continúa la línea de la TER con atención particular en las organizaciones, evidente en su libro $A$ behavioral model of rational choice, publicado en 1955. Su tesis y argumentación ha provocado que sea considerado como uno de los padres de la IA, al poner sobre la mesa las limitaciones cognitivas del comportamiento humano en la solución de problemas, así como las posibilidades de la acción inteligente.

El autor apunta, desde la economía tradicional, que el hombre siempre se ha visto a sí mismo con una función económica y que en su búsqueda y realización ha sido irracional, puesto que asume que cuenta con información relevante y con las herramientas necesarias para calcular, entre las distintas opciones de acción que tiene a su disposición, aquellas que le permitan alcanzar el punto más alto en la escala de sus preferencias. La discusión no se centra solamente en el hombre económico, sino que avanza teóricamente hacia el hombre administrativo. Esta perspectiva, relacionada con los procesos cognitivos y de toma de decisiones, ayudaría a mantener el rumbo de la elección óptima (Simon, 1955).

Simon (2007) plantea que ningún individuo al momento de elegir entre alternativas puede escapar de la influencia de las características del ambiente ni de las interrelaciones que resultan de su interacción. De esta manera se posiciona como el punto central de su argumentación al subrayar el valor de la información que el individuo posee para llevar a cabo su elección. Asimismo, señala entre las restricciones principales: a) el conjunto de las alternativas entre las cuales puede decidir, b) las relaciones que determinan un objetivo satisfactorio, en función de las alternativas posibles, así como c) la jerarquía de los costos.

En este contexto, el contrato entre las partes resulta de una conducta maximizadora que recibe influencia de la asimétrica disponibilidad de información de cada una de ellas, a la par del poder de negociación y de las oportunidades de "hacer trampa o de engañar", lo que produce una especie de racionalidad limitada o acotada (Simon, 2007, p. 55). 
En la lectura de los trabajos de Simon $(1955 ; 2007)$ puede seguirse que la aportación principal a la TER fue explicar las limitaciones y hacer visible la incertidumbre que tienen los individuos al racionalizar sus decisiones, proceso en el cual confluyen percepción, intuición, contenidos de la información y el comportamiento racional y su evaluación, elementos que conllevan un aprendizaje. Esto lleva a la idea de que la conducta de elección racional que controla y es considerada como óptima, tal como lo propuso Weber, puede adaptarse y encontrar un equilibrio entre los límites máximo y mínimo; elegir no la mejor opción sino una buena opción, lo que se entendería como un nivel satisfactorio (Simon, 1955). Como puede intuirse, en el núcleo de sus argumentos se encuentra la información, misma que permite trazar un mapa de las opciones que interfieren en el proceso de toma de deciones.

Por su parte, Elster (2005) propone una nueva perspectiva de la TER a partir de la tesis de que la acción social no solo depende de la racionalidad. Los distintos efectos que examina ligados con los mecanismos microfundamentos, le permiten adentrarse en un modelo teórico -por un lado prescriptivo y por otro cooperativoque contribuye al análisis del orden social, otorgándole un poder explicativo a las relaciones causales, en las que intervienen emociones, creencias, deseos, valores, percepción y presión social, constituidas como las normas sociales que motivan la acción, elementos explicativos del comportamiento individual pero también colectivo. Por lo tanto, el enfoque prescriptivo de la TER resulta de esta posibilidad de alternativas entre las cuales el individuo crea expectativas en interacción con sus influencias culturales, elige su acción y toma sus decisiones. Las normas sociales del presente se visualizan como guía de la acción.

Siguiendo la ruta y en el intento de establecer el vínculo entre la TER y los arreglos institucionales, Douglas North (1971) hace hincapié en que estos surgen a través de tres vías: por voluntad, por coerción (gubernamental) y en una combinación de ambas. En 1994 señala que el desempeño de los agentes sociales no puede ser explicado sino en el contexto de la existencia de restricciones formales (como leyes, reglas y constituciones) o informales (normas, ritos, rutinas, procesos y principios) que actúan como puntos de apoyo en la toma de decisiones.

North (1971, p. 360) concibe a las instituciones como "las restricciones que los humanos idean para dar forma y estructura a sus interacciones", lo que se traduce a una racionalidad instrumental. De esta manera es que se resalta la importancia de llegar a arreglos institucionales, los cuales determinarán un marco de actuación so- 
cialmente construido. Son definidos por Ostrom (2015, p. 109) como: el conjunto de reglas en uso que se aplican para determinar quién tiene derecho a tomar decisiones en cierto ámbito, qué acciones están permitidas o prohibidas, qué reglas de afiliación se usarán, qué procedimientos deben seguirse, qué información debe o no facilitarse y qué retribuciones (o amonestaciones) se asignarán a los individuos según sus acciones."

Destaca que los agentes sociales siendo racionales en lo individual tendrían que ser racionales también en lo colectivo, sobreponiendo la búsqueda del bien común sobre el bien particular. En esto coincide con el llamado efecto de derrame que explica Elster (2005). Ostrom (2015) insiste en que si los miembros de un grupo comparten un objetivo e intereses y todos ellos obtendrían un beneficio si se consigue, en un sentido lógico todos ellos enfocarán sus esfuerzos individuales en alcanzarlo.

Figura 2. Evolución de la TER

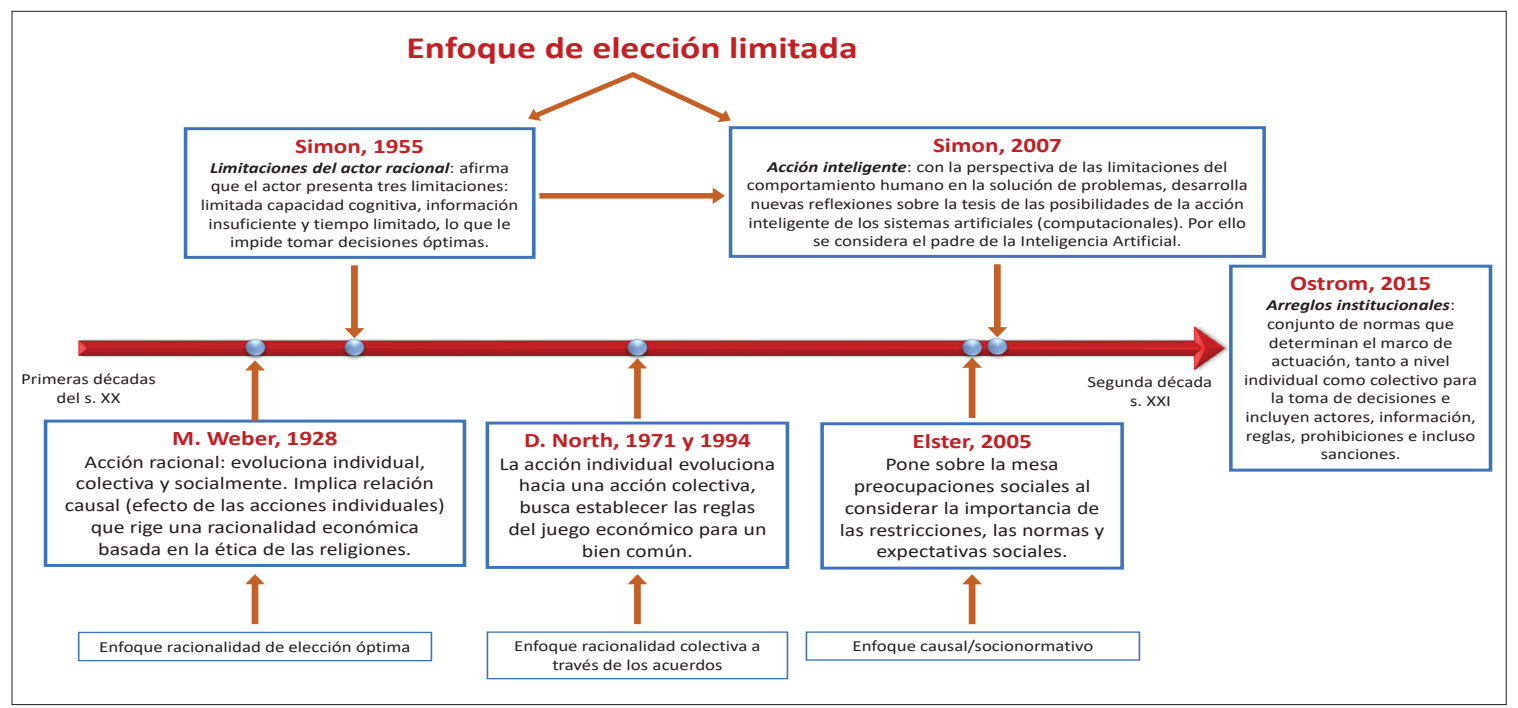

Fuente: Elaboración propia a partir de Weber (1928), Simon (1955; 2007), Elster (2005), North $(1971 ; 1994)$ y Ostrom (2015).

Se ha identificado que los individuos poseen distintos tipos de racionalidad, aun en el tiempo actual en que sus premisas han evolucionado. La racionalidad maximizadora de numerosos actores económicos hoy en día se compagina con la racionalidad limitada por la irregular información que se posee (incluso en su interacción a nivel de gestores del territorio, habitantes, empresarios o turistas) y porque suelen 
pasarse por alto las normas sociales presentes en la escala local de los destinos turísticos.

De igual forma, es posible identificar una racionalidad práctica e interactiva entre los turistas, en particular con el uso de las aplicaciones digitales, lo que entra como complemento también de una racionalidad maximizadora -económica-, al buscar el mayor beneficio posible de su experiencia turística. En el mejor de los casos buscarán la opción que mejor satisfaga sus intereses, al verse en la necesidad de hacer compatibles la información sobre el destino, el tiempo disponible y el costo de los servicios/ingreso personal (racionalidad limitada - satisfacción). Los desarrolladores tecnológicos y formadores educativos resuelven a partir de una racionalidad tecnológica, tal como lo apuntó de forma temprana Weber (1928). En síntesis, son los distintos tipos de racionalidad que permiten comprender la acción y los procesos de toma de decisiones en el campo del turismo.

La IA se basa en el diseño de algoritmos que ayudan a los usuarios en el proceso de toma de decisiones, incluso en ambientes de incertidumbre. Sin embargo, aunque resulten a primera vista herramientas rápidas y cómodas, propician un ambiente carente de control social y transparencia, lo que hace poco visible el análisis de la intencionalidad de las decisiones y, a su vez, limita acciones de gestión y planeación y conduce a escenarios poco precisos.

No obstante, los diseñadores y propietarios de las distintas plataformas digitales y empresarios, al tener el control de la información y de los impactos sociales y económicos que pueden generar las decisiones, resultan mejor equipados para llevar a cabo una elección óptima, lo que les permite maximizar sus beneficios. Estos acercamientos sobre la TER, la acción de los agentes y los arreglos institucionales, resultan acordes con el objetivo y la hipótesis de este trabajo, puesto que tienen el potencial como plataforma explicativa, la responsabilidad respecto de la información y de la actuación de los agentes del turismo involucrados en el proceso de toma de decisiones, pasando así por la racionalidad económica, práctica o interactiva hacia la evaluación causa - efecto de sus acciones, tanto en un sentido individual como colectivo.

En este sentido, el diálogo en la escala local, de inicio voluntario y con influencia de las restricciones informales, tiene el potencial de transformarse en restricciones formales, con lo cual los acuerdos resultantes adquieren un carácter mixto, según lo expone North $(1971 ; 1994)$. Se propone entonces transitar hacia una racionalidad 
responsable con un efecto de permeabilidad a todos los agentes del turismo y a todas las escalas territoriales. Son las normas sociales, según lo expone Elster (2005), que se definen y hacen visibles en la escala local y permiten el análisis de cuestiones sociales, las que determinan en el largo plazo y de forma significativa el desempeño económico y social de la actividad turística.

\subsection{La importancia de la escala local para llegar a los arreglos institucionales}

En el ámbito geográfico, es una convención que el territorio es determinante de las relaciones socioculturales, económicas y ambientales que resultan de distintas formas de apropiación del territorio y de la participación-acción en los procesos que ocurren en el plano espacio-temporal; no es, de forma ingenua como muchos aún suelen pensar en el campo del turismo, el simple escenario donde se desarrollan las prácticas turísticas. Agentes públicos y privados, organizaciones gubernamentales, no gubernamentales y empresariales, población local y hasta los turistas, ponen en juego sus intereses, normas y valores, intenciones y sus racionalidades, lo que configura un esquema de poder en el cual se vuelve necesaria la información, la negociación y la sinergia.

Con frecuencia se pierden de vista las interrelaciones en el turismo, se ignora la conexión entre su práctica y el resto de las actividades que en el territorio se desempeñan y se difuminan los agentes, sus intereses e intencionalidades, su racionalidad. Como agentes sociales las empresas suponen un espacio económico en el que interactúan, del que dependen y, a su vez, al que afectan y hasta controlan. Considerar la perspectiva territorial en las implicaciones de la RTD significa identificar a los distintos grupos de implicados y sus claves de organización histórica incluso, más allá de las prácticas turísticas.

Mientras que algunos teóricos sobreponen el poder homogeneizador de la globalización económica sobre el potencial de la escala local, otros insisten en la fortaleza de las respuestas que emanan de los ámbitos locales. La pandemia por el COVID-19 puso en relieve las posibilidades y el sentido de lo local para responder a una problemática global y el sector turístico no es la excepción: la reactivación de la actividad ha centrado sus estrategias en esta premisa.

No obstante, hablar de la escala o del enfoque local no exige coincidir con una porción del territorio específicamente delimitada, su definición obedece en mayor me- 
dida a las relaciones sociales, ambientales y económicas, así como a las interacciones culturales. En esta discusión puede agregarse que la primera característica se refuerza a partir de las decisiones y la racionalidad de los agentes locales; la segunda se manifiesta en la capacidad real de ofrecer respuestas y conseguir lo que mejor convenga al territorio, algo que sea satisfactorio para todos, la puesta en común de objetivos, intereses y recursos individuales transformados en colectivos, tal como lo señala Ostrom (2015), mientras que la tercera orienta las respuestas, propiamente dichas, en la forma de los arreglos resultantes.

La existencia de una sociedad local constituye un condicionante para instituir la responsabilidad en un sentido amplio, debido a que esta no puede darse si carece de dicha sociedad local que la adopte como una acción transversal y como parte de un proyecto común, inclusive si se considera la cuestión de la globalización en el campo del turismo o la presencia de agentes originalmente extralocales.

De esta forma, los agentes locales del turismo tienen la responsabilidad de identificar las transformaciones producidas por el uso de esta disrupción tecnológica, de interpretarlas, de orientar el aprendizaje y traducir el nuevo conocimiento en procedimientos operativos y acoplados en el entorno económico, cultural, comunitario y político local: de guiar el cambio. Pero este proceso no siempre resulta en consensos, por el contrario, es tan frágil que obliga a la construcción permanente de un proceso de negociación. De ahí la importancia de considerar la escala local y la racionalidad de los agentes sociales para instituir la RTD.

Un agente bien informado y responsable es consciente de que la acción individual abre la posibilidad a la reconstrucción de la realidad local, lo que permite establecer nuevas opciones o vías para el desarrollo orientadas a detonar la razón colectiva para la construcción compartida y la responsabilidad territorial con varios vértices: consenso social, conocimiento científico y poder político colectivo, que son elementos clave para el desarrollo de pactos y contratos sociales inteligentes que afronten los nuevos retos impuestos por la era digital a los territorios o destinos turísticos.

\subsection{La definición propuesta de RTD}

Desde el ejercicio plural de la filosofía, pensadores como Platón, Jonás, Descartes, Kant e incluso Weber, entre otros, han abordado y acotado la cuestión según el campo de aplicación, pero las raíces continúan dando soporte a los debates actuales. Con base en la fundamentación que Misseri (2012) distingue desde el pensa- 
miento de los griegos, pero con énfasis en la influencia de Platón y Jonás, señala que el concepto remite a la capacidad de responder por los actos principalmente dirigido hacia los grupos de poder, hacerse cargo de las consecuencias de la acción, pero darse cuenta de esto supone un ejercicio de introspección que lo lleva al sentido racional; se agregaría que supone un estado de consciencia potencialmente receptivo de las preocupaciones sociales.

Por su parte, el economista Howard Bowen, considerado como el padre de la Responsabilidad Social Empresarial (RSE), publica en 1953 su libro Social responsabilities of the businessman en donde de forma temprana define la responsabilidad social como los acuerdos entre los empresarios para establecer las políticas corporativas que les permitan tomar decisiones, en un marco de actuación fincado en valores socialmente deseables, en un momento histórico en que los cambios sociales exigían nuevos métodos productivos que pudieran vincular el interés individual de los hombres de negocios con el interés social de maximizar la producción y el progreso económico, durante y después de la II Guerra Mundial (Acquier et al., 2011).

Así, trasladar el discurso de la responsabilidad social al campo del turismo supone la necesidad de explicar de qué forma van a entenderse los arreglos o acuerdos institucionales. Estos se definen como el conjunto de normas, reglamentos y políticas que se discuten y pactan entre los agentes sociales del turismo económicos, gubernamentales, políticos, profesionales, habitantes, desarrolladores digitales, usuarios y propietarios de las plataformas y productos digitales, para que en el marco de actuación del sector, contribuyan a consolidar un ambiente de legitimidad, justicia y equidad social (Ostrom, 2015). Establecen el ámbito de referencia en el cual se desarrolla el proceso de toma de decisiones y les ofrece las posibilidades de retribución o penalización según los efectos producidos por sus decisiones.

Con los referentes teóricos heredados y con la intención de ofrecer una primera definición para dar comienzo la discusión, se considera que la RDT es el resultado de un arreglo institucional que se finca en:

- El contexto del turismo en la era digital: el turismo 4.0 y su trayectoria futura hacia un reposicionamiento de la ética y la responsabilidad.

- Considerar a los distintos agentes del turismo y sus normas sociales, valores, intereses y racionalidades individuales y colectivas; en el marco del sistema local se pone en acción el poder de la negociación y la sinergia: fortalece la po- 
sibilidad de llegar a acuerdos, se constituye como un soporte para la toma de decisiones, fundamentado en principios éticos.

- Hacer conciencia de forma reflexiva respecto de las posibilidades de uso benéfico de la IA para el desarrollo del turismo, pero también de los riesgos que suponen a las empresas, a las personas y al territorio, enfoque causa y efecto.

- Conciliar un marco legal que permita dar certidumbre a las acciones y decisiones resultantes a largo plazo, fundamentalmente en el campo de la protección de datos personales y del patrimonio del usuario. Dicho marco deberá estar dirigido igualmente a la mejora de los servicios de salud, seguridad, comunicación, movilidad, gestión de servicios públicos y medio ambiente, considerando el impulso al talento, la innovación y a aquellos aspectos que optimizan la calidad de vida de las comunidades. Lo anterior son condiciones esenciales para el desarrollo de los territorios turísticos, por lo cual es necesario que se hagan visibles en la política pública.

\section{Notas para reflexionar en el campo del turismo. La hiperconexión y la responsabilidad}

Mucho se ha discutido sobre el futuro del turismo y la supuesta "nueva normalidad"; no obstante, los factores de recuperación apuntan a escalas territoriales menores, destinos de proximidad y cercanías, sobre todo en el ámbito rural. La famosa premisa de "pensar globalmente y actuar localmente" (Think global, act local), así como las oportunidades de trabajar y reforzar la escala local, son más visibles y mejores que nunca; de ahí que las posibilidades de establecer compromisos institucionales en escalas menores y que estos posteriormente avancen hacia otras escalas territoriales están puestas sobre la mesa (Geddes, 1915; Organización de las Naciones Unidas, 1992).

La actividad turística tiene como característica esencial su espacialidad. La interacción de múltiples esferas y escenarios políticos, ambientales, sociales, económicos y hasta tecnológicos, no hace más que reiterar que se trata de una actividad geográfica. Sin embargo, el turismo también se explica por su apertura y globalidad. Su ejercicio, aun cuando se manifieste en el nivel local, representa interacciones en y desde distintas escalas territoriales.

Las predicciones mundiales respecto al crecimiento de la actividad eran más que triunfalistas. En el documento Panorama OMT del Turismo Internacional (Organiza- 
ción Mundial del Turismo, OMT, 2017) se hablaba del creciente y sostenido flujo de turistas internacionales y era cuantitativamente cierto, las proyecciones para el año 2030 apuntaban como meta los 1.8 billones de turistas. Durante el año previo a la pandemia, este organismo reportó un movimiento de turistas internacionales superior a los 1.46 billones, la posición de México mejoró en el ranking mundial, al pasar del $8^{\circ}$ al $7^{\circ}$ lugar en el mismo año. La escalada del número de viajeros se había presentado de forma constante, acelerada e ininterrumpida, hasta que llegó el COVID. Esta situación afectó de forma dramática la industria de los viajes y el turismo, con una estrepitosa caída que en términos generales significó el descenso de un 70\% durante el 2020.

Lo anterior se sujeta a distintas lecturas si se afina la escala: la región asiática registró pérdidas de $96 \%$ mientras que en la región de América se redujo el movimiento un 79\%, esto con la reapertura de algunos destinos a partir del último trimestre de 2020, segmentado en los servicios de líneas aéreas, hoteles, cadenas de restaurantes e incluso en empresas de recorridos turísticos, todas ellas inmersas en esta disrupción tecnológica. Un comunicado de prensa de la OMT (2021) señala una pérdida acumulada en el primer trimestre del 2021 del orden del $85 \%$.

Para México la situación también pone en evidencia una caída importante, pero atenuada discursivamente al señalarse una mejor posición del país en el ranking mundial, un tercer lugar durante el 2020 -aunque cabe decir que los viajes hacia México nunca se han suspendido, un tema para discutirse desde la responsabilidad-. A finales de 2020, se vaticinaba un escenario altamente positivo para 2021: se alcanzarían los 42.7 millones de turistas internacionales (Sectur, 2020). Esta expectativa tuvo que reformularse con los ajustes del primer trimestre de 2021; hoy se visualizan tres escenarios posibles: optimista (33.1 millones), conservador (30.4 millones) y pesimista (25.2 millones) (Sectur, 2021).

En este contexto aparecen numerosas propuestas que abren la posibilidad de viajar sin salir de casa: redes sociales, turismo virtual y de realidad aumentada, aplicaciones y gadgets de idiomas para viajeros, de fotos artísticas (filtros), de reservaciones de vuelos o entradas a sitios turísticos, teatros o eventos virtuales, de gestión de reservas; de geolocalización de lugares turísticos, de mapas y movilidad en destinos, aplicaciones y plataformas de hospedaje alternativo a la modalidad hotelera y como resultado propio de la pandemia el binomio trabajo + vacaciones se potencia: bleisure (Entono Turístico, 2016; Forbes, 2020). 
Es necesario sumar nuevas medidas de control a las ya existentes, principalmente en el ámbito de la salud, por ejemplo: pagos con tarjetas digitales, pasaportes de vacunación ligados a una red mundial de datos; tecnología de reconocimiento facial o la robotización de los servicios turísticos para evitar el contacto personal, así como sensores que miden la calidad del aire, agua o su temperatura e incluso puntos de saturación de personas con sensores de calor o ruido. Cada nueva aplicación solicita que se acepten o rechacen las opciones del uso de cookies'.

Interesa destacar el tema de la confianza o desconfianza que el usuario deposita en la organización que recabará los datos, la garantía de anonimato le permitirá reducir riesgos y asegurar su uso ético. Esto lleva a hablar de la RSE, pero también de la responsabilidad de los agentes gubernamentales, quienes "deberían" garantizar mecanismos formales de privacidad desde el diseño, con lo que se evitará un uso posterior de los mismos con fines distintos a los que fueron recabados. Como estrategias restrictivas deberían funcionar, al menos para las empresas que se establezcan en el ámbito local.

Sin embargo, en el campo del turismo los propios turistas se constituyen como los principales alimentadores, incluso desde antes de comenzar el viaje. La práctica turística de los viajeros contemporáneos se caracteriza por la inmediatez, una urgencia por compartir la experiencia en tiempo real: ya en el aeropuerto, a punto de comenzar mi viaje... foto en instagram, aquíllegando al hotel... check in, les comparto este delicioso platillo... foto, en la fila para entrar al palacio de... selfie, todo ello le permite personalizar los contenidos, una vez que se busca información sobre un destino o producto turístico comienzan a llegar más y más datos relacionados, la IA ha aprendido cuáles son sus preferencias.

Esta digitalización de la vida cotidiana que convierte lo privado en público es lo que marca la paradoja. Al exponer su privacidad los turistas la entregan como mercancía a las empresas de desarrollo tecnológico. La pandemia por el COVID-19 ha incrementado notablemente los riesgos, a tal grado que se estima un empoderamiento de la racionalidad tecnológica. Lo anterior lleva de vuelta a la necesidad de generar espacios de comunicación en los cuales se discutan las preocupaciones del sector y de los agentes y se propicien mecanismos que permitan tranquilidad en el proceso de la toma de decisiones: los arreglos institucionales.

1 Paquete de datos que un navegador web almacena de forma automática en la computadora de un usuario cuando este visita una página web. 


\subsection{Entonces: ¿cuál es el camino a seguir por los agentes sociales para construir los arreglos necesarios?}

Tanto líderes mundiales como los mismos desarrolladores tecnológicos que han marcado el rumbo reciente de la humanidad ya reflexionan y llaman la atención al respecto. Klaus Schwab, fundador del Foro Económico Mundial, rotula que los avances tecnológicos no han sido debidamente tomados en cuenta en un marco legal, lo que puede conducir a una "ruptura del contrato social entre los gobiernos, las empresas y los ciudadanos; crearse nuevas reglas del juego, reposicionar el sentido ético y establecer controles y equilibrios que permitan recuperar la justicia y la equidad, la seguridad y confianza, así como la propiedad intelectual" (Schwab, 2016, p. 60). La estrecha colaboración entre los distintos agentes del turismo es fundamental, sus intereses deben formar parte del diseño de la agenda pública de asuntos turísticos, pues esta constituye la guía de acción.

Otra preocupación viene de la Organización para la Educación, la Ciencia y la Cultura de las Naciones Unidas (UNESCO, 2020). Este organismo reitera la necesidad de contar con un instrumento normativo sobre la ética en la IA, el tema se presenta como un reto crucial pues deberá establecerse como un acuerdo, sujeto de negociación entre 193 países miembros, ejercicio en el que participan 155 expertos y miembros de la sociedad civil con lo que pone la mirada en la escala local. Existe el temor de que la tecnología tome decisiones por los humanos, lo que provoca la violación de sus derechos y una creciente manipulación.

Tal es la situación que la OMT declara el tema del Día Mundial del Turismo en 2018: el turismo y la transformación digital, con un llamado a tomar en cuenta el desafío de hacer compatible el crecimiento del turismo con los paradigmas de la sustentabilidad y la responsabillidad, dinámica en la que los avances digitales y la innovación juegan un papel importante (OMT, 2018).

En el sitio INVAT-TUR, el director de Inteligencia Turística de la Comunitat Valenciana, Mario Villar (2021, párr. 2) diserta acerca de una iniciativa que se presenta desde la Comisión Europea en la cual se establece un nuevo marco regulatorio para la IA, argumentando que el turismo no puede permanecer ajeno a la revolución que supone su avance: "Europa no quiere que se vulnere la seguridad y los derechos fundamentales de la ciudadanía y las empresas".

En el mismo orden de ideas, la vicepresidente de la Comisión Europea, Margrethe Vestager declaró que: "en inteligencia artificial, la confianza es imprescindible, no 
un lujo" (citada en Villar, 2021, párr. 2). De esta iniciativa se recogen los Códigos Éticos que descienden desde ambientes internacionales como la OMT, hasta las comunidades de menor escala territorial, casos documentados que combinan el uso de IA y ética en el campo turístico y de los que se desprenden las siguientes reflexiones (Villar, 2021):

a) Establecer cuándo es necesaria la acción y supervisión humana sobre los algoritmos.

b) Evaluar la solidez técnica y seguridad de los modelos de inteligencia artificial y su resistencia a ciberataques. La gestión de la privacidad y los datos de los viajeros, actualmente son unos de los riesgos principales.

c) La explicabilidad de los algoritmos. Garantizar la diversidad, no discriminación y equidad: asegurar que los datos que se utilizan en el modelo no arrastran sesgos.

d) Valorar la contribución de la IA a alcanzar el objetivo de crear bienestar social y ambiental. La rendición de cuentas.

En el contexto de la era digital, la responsabilidad adquiere un carácter preventivo, le corresponde conciliar en los términos y con las características que los agentes sociales del turismo acuerden en el ámbito local. Esto remite a uno de los pilares de la sustentabilidad: prevenir en vez de corregir, pero también a la necesidad de una actuación de contenido ético.

En particular, se trata de razonar respecto al deber ser de la actuación de los agentes del turismo en entornos digitales, para colaborar en la resolución de los problemas del territorio que desempeña la función turística, con el objetivo de construir una agenda que ofrezca respuestas a los requerimientos sociales, según lo expresa Elster (2005). A partir de lo anterior y en el intento de cumplir con el objetivo de este trabajo, se propone que el diseño de estos arreglos puede comenzar con las siguientes acciones:

a) Definir la escala territorial de trabajo de funcionalidad de los destinos turísticos. ¿Cuál será el ámbito considerado como lo local? ¿los agentes del turismo, toman en cuenta el marco socio-territorial en el que interactúan? ¿son capaces de identificar las normas sociales que definen la acción, según lo propone Elster? 
b) Identificar los agentes del turismo que interactúan en el destino turístico y definir su racionalidad, acorde a los llamados de la TER. Esto conlleva un ejercicio de reflexión respecto de los límites de su acción ante valores superiores como la equidad, justicia, desarrollo, ser humano, ambiente o salud, según los principios éticos de distintas organizaciones.

Establecer quiénes son los agentes sociales del ámbito local que deben estar en este diálogo de carácter transversal: ¿quién tiene derecho y obligación a participar de los procesos de innovación en escenarios digitales del turismo? ¿Cuáles son las preocupaciones sociales locales que se ponen sobre la mesa de diálogo? Introducir un enfoque prescriptivo y de cooperación.

Con el propósito de identificar las racionalidades de los agentes del sistema turístico se ha realizado la categorización de los distintos agentes sociales, resultando cinco grupos: 1) gestores públicos locales, 2) empresarios (turísticos y desarrolladores tecnológicos), 3) turistas, 4) población local y organizada y 5) comunidad académica, para cada uno de ellos, es necesario revelar su racionalidad, cuestionar y reflexionar colectivamente sobre lo que involucra la RTD, los temas y las tareas implicadas para llegar a los acuerdos necesarios.

Reflexionar respecto a ¿cuáles son las oportunidades y los riesgos de la IA en el turismo y en el caso particular del territorio turístico? ¿cuál es el marco legal y los mecanismos de evaluación, control y sanción requeridos para eliminar la falta de ética de algunos agentes sociales y el uso indebido de datos personales? Ilamando a evitar la racionalidad económica maximizadora.

c) Parte importante de los acuerdos es determinar ¿quién garantiza o asume la responsabilidad, la privacidad, seguridad, integridad y protección de los datos? y ¿cómo se determinan las responsabilidades respecto al uso de herramientas digitales desde el territorio turístico? Lo anterior desde una perspectiva causa-efecto. Asimismo, ¿de qué forma se puede alimentar una política pública de responsabilidad turística digital en cada territorio turístico? con opciones que satisfagan a los distintos grupos: negociación y consenso.

Identificar las estrategias que permitirán reducir la incertidumbre sobre el futuro, principalmente fundamentadas en un acceso a la información. Y desde la formación y educación turística ¿cuáles son las plataformas educativas y cognitivas que deben ser impulsadas para hacer frente a la dinámica digital en el turismo? 
Como consecuencia de la interacción actor-racionalidad-principios, surgen los lineamientos para incidir en la conceptualización de la RTD y en su diseño como institución. Lo que hace cada quién para conseguir conciliar el capital social y la ética. A partir del diálogo y de debatir estos y otros cuestionamientos que pudieran resultar, será posible exponer los principios de la RTD, la forma que esta debe tomar para que sea aceptada y asumida por los agentes sociales del turismo, lo que la llevará al grado de institución. Como en distintos asuntos ligados con el turismo y otras actividades, la escala local ofrece las posibilidades de cooperación, de acción conjunta, de otorgar rumbo y validez a una estrategia de trabajo.

Ante el escenario mundial actual (pandemia COVID-19), el tema de la sustentabilidad invita a debatir el futuro del turismo en un ambiente menos congestionado, con usos distintos del territorio y entornos digitales más socializados y de contenido ético. Se hace énfasis en un mayor alcance del patrimonio territorial "turistizado", lo que lleva a la necesidad de incorporar principios derivados de paradigmas sobre el desarrollo, particularmente el de enfoque local.

\section{Conclusiones}

Diseñar una institución de RTD es un proceso complejo y de largo plazo, porque el tema de responsabilidad digital es relativamente "nuevo" y no todos los agentes sociales tienen el mismo nivel de conocimiento respecto a las implicaciones de la inteligencia artificial. El mosaico de agentes involucrados y los diferentes niveles de actuación y racionalidad, requieren de espacios de discusión diversos y diálogos en horizontes y ambientes heterogéneos.

Las diversas aportaciones de la TER que fueron revisadas previamente muestran un camino por el cual transitar. Es trascendental que los agentes sociales del turismo no solo consulten la mayor cantidad de información, sino que pongan sobre la mesa lo que esperan de las instituciones, es decir, sus expectativas, así como asirse al contexto social en el que interactúan y su percepción, de forma que puedan tomar las decisiones como agentes e identificar la relación causal de sus elecciones.

En este punto los arreglos institucionales consolidan un ambiente de legitimidad, justicia y equidad en la sociedad con la que interactúan, ya que sus esfuerzos se dirigen tanto a la protección de datos personales como del patrimonio del usuario, así como a la mejora de los servicios de salud, seguridad, comunicación, mo- 
vilidad, gestión de servicios públicos y medio ambiente, en el impulso del talento, la innovación y en todos aquellos aspectos que mejoran la calidad de vida de las comunidades. Se trata de un llamado a una racionalidad extendida o múltiple, que supere distintas racionalidades que resultan más acotadas para el desarrollo de los territorios turísticos.

La importancia de que los agentes del turismo cuenten con pactos de responsabilidad en entornos digitales, estriba en un ejercicio potencial para repensar su racionalidad y rejerarquizar sus objetivos, así como tomar conciencia de los efectos de sus decisiones, es decir, regular sus acciones. Los agentes encargados del diseño y producción de plataformas digitales, adquieren un "carácter moral", por lo tanto están obligados a actuar con restricciones institucionales de responsabilidad y sujetar sus acciones al escrutinio social. Es preciso poner límites al negocio de la gestión de la privacidad: pugnar por una racionalidad responsable.

La propuesta de transitar hacia la construcción de arreglos institucionales lleva al objetivo de reducir la búsqueda de la elección óptima (basada en una racionalidad de interés individual) y, como señala Simon (1955; 2007), priorizar la racionalidad práctica e interactiva del sistema turístico y de territorio, a favor de estrategias que busquen el uso responsable de la tecnología. Sin embargo, aunque se sostiene que la escala local ofrece el ambiente ideal, en esta etapa solo hubo un acercamiento desde el nivel teórico conceptual y no directamente con grupos de algún territorio en particular, lo que al mismo tiempo que limita avanzar en la reflexión, presenta una vía de trabajo a futuro.

La idea en este momento no es tratar de incidir en la gran escala de las empresas tecnológicas globales, lo que efectivamente resulta muy complicado desde este ámbito; significa establecer acuerdos sobre el marco normativo que protejan los derechos de los usuarios, promuevan su privacidad y limiten su manipulación; primero para la actuación gubernamental y segundo para las empresas locales, así como impulsar un mecanismo de aprendizaje social que haga que las personas actúen responsablemente a nivel global, en el sentido equiparable a lo que señalan Ostrom (2015) y Elster (2005).

Los diálogos que se construyen en torno a la dinámica de la RTD requieren de un carácter transversal, en un sentido horizontal, pero también vertical y se abre la oportunidad de salvar la línea que separa un ejercicio voluntario de uno normativo, obligatorio para todos, en colectivo, como lo manifiesta North (1971). 
La eficiencia de la institución de RTD, tal como lo refiere North $(1971 ; 1994)$ debe evidenciarse en la reducción de la incertidumbre que provoca el desconocimiento sobre cómo se llevan a cabo los procesos y cuáles son los alcances del desarrollo tecnológico que se utiliza actualmente y hacia el futuro en la actividad turística, se pone en relieve el valor de la información, de acuerdo con los planteamientos de Simon $(1955 ; 2007)$.

Tanto el diseño como el cumplimiento y la consolidación de los arreglos institucionales, así como su trascendencia en el largo plazo, se sujetan a tres características básicas: confianza, reputación y reciprocidad, lo que habla de la existencia de un capital social fuerte que puede estar presente en los destinos turísticos (escala local). De la misma forma en que el turismo actúa y tiene efectos a lo largo de distintas esferas, obliga a repensar el papel de la tecnología y la ética, por la forma en que impacta en otros sectores más tradicionales y preexistentes que el turismo.

Por supuesto que el medio formativo y profesionalizante, tanto en el turismo como en el campo del desarrollo tecnológico, legislativo y de gestión pública (gobernanza), deberá tener un lugar en la mesa de diálogo y negociación: se precisa que estos asuntos formen parte del diseño de políticas públicas del turismo, con vigilancia legal y normativa que concuerde con el ritmo del avance tecnológico.

\section{Referencias}

Acquier, A., Gond, J. P. y Pasquero, J. (2011). Rediscovering Howard R. Bowen's legacy: the unachieved agenda and continuing relevance of social responsibilities of the businessman. Business \& Society, 50(4), 607-646. http:// dx.doi.org/10.1177/0007650311419251

Aguilar, L. (2020, 24 de junio). Modernidad y Racionalidad. Centenario de la muerte de Max Weber [YouTube]. Centro Latinoamericano de Administración para el Desarrollo. https://bit.ly/3BbDJpP

Arencibia, F., Peña, B. y Pardo, A. (2020). El falso conteo de las revoluciones industriales: de la 1 a la 5 . Productividad y mano de obra. Fin del paradigma, comienzo de la ética. Aglala, 11(1), 95-106. https://bit.ly/3hJQyjp

DataTur. (2015). Documentos de investigación estadística y económica. https://bit. ly/3ks6Wqo 
Diario Oficial de la Federación (DOF). (2010, 5 de julio). Ley federal de protección de datos personales en posesión de los particulares. https://bit.ly/36LiHjC

Diario Oficial de la Federación (DOF). (2021, 16 de abril). Decreto por el que se reforman y adicionan diversas disposiciones de la Ley Federal de Telecomunicaciones y Radiodifusión. https://bit.ly/2VRHsIY

Elster, J. (2005). A favor de los mecanismos. (Nicolás Loza Otero, Trad.) Sociológica, 19(57), 239-273. (Obra original publicada en 1998). https://bit.ly/3kqwPad

Entorno Turístico. (2016). Bleisure. https://bit.ly/3hJOckz

Forbes. (2020). Bleisure: la combinación perfecta entre trabajo y placer. https://bit. ly/3im5jYI

Foro Económico Mundial (WE Forum). (2021). Berners-Lee, padre de la web, lanza una campaña por una internet mejor. https://bit.ly/3enseSk

Geddes, P. (1915) Cities in evolution. https://bit.ly/36Hz1lG

Infolat. (2021, 20 de junio). App de Google para Android facilitó el robo de datos privados de sus usuarios. https://bit.ly/3epbZEi

INVAT-TUR. (2021, 11 de junio). Inteligencia artificial en el sector turístico [archivo digital]. https://bit.ly/3rdnsMq

Misseri, L. (2012). La responsabilidad utópica: Platón y Jonas. Utopía y Praxis Latinoamericana, 17(56), 59-69. https://bit.ly/3kqOAWN

North, D. (1971). Institutional change and economic growth. The Journal of Economics History, 31(1), 118-125. https://bit.ly/2UlHUif

North, D. (1994). Economic performance through time. The American Economic Review, 84(3), 359-368. https://bit.ly/3igZ4FM

Organización de las Naciones Unidas (ONU). (1992). Declaración de Río sobre el medio ambiente y el desarrollo. https://bit.ly/2UmSkOt

Organización Mundial del Turismo (OMT). (2017). Panorama OMT del Turismo Internacional. https://doi.org/10.18111/9789284419043

Organización Mundial del Turismo (OMT). (2018, 26 de septiembre). Celebración oficial del Día Mundial del Turismo 2018. Turismo y transformación digital. https://bit.ly/3hHvdqT 
Organización Mundial del Turismo (OMT). (2021, 2 de junio). El turismo cae un 83\%, aunque la confianza mejora lentamente [comunicado de prensa]. https://bit. ly/3wLpkgv

Organización de las Naciones Unidas para la Educación, la Ciencia y la Cultura (UNESCO). (2020, 17 de septiembre). La UNESCO da un gran paso hacia el primer instrumento normativo sobre la ética de la IA. https://bit.ly/2VQkG4d

Orlowski, J. (Dir.) (2020). El dilema de las redes sociales [documental]. https://bit.ly/ 36Fv54P

Ostrom, E. (2015). El gobierno de los bienes comunes. La evolución de las instituciones de acción colectiva. Fondo de Cultura Económica.

Reportur.mx. (2019). Masivo robo de datos de hoteles latinos y agencias como Booking. https://bit.ly/3ik7trL

Schwab, K. (2016). La cuarta revolución industrial. World Economic Forum y Debate. https://bit.ly/3kyXH7Q

Secretaría de Turismo (Sectur). (2020, 27 de diciembre). Presenta Miguel Torruco expectativas de la industria turística para 2021 [comunicado de prensa]. https://bit.ly/3xMiqJm

Secretaría de Turismo (Sectur). (2021, 18 de enero). Expectativas de la industria turística para 2021, considerando las medidas implementadas por el gobierno de EU [comunicado de prensa]. https://bit.ly/36DwVDr

Simon, H. (1955). A behavioral model of rational choice The Quarterly Journal of Economics, 69(1), 99-118. https://bit.ly/3Bb1Cxz

Simon, H. (2007). Organizations and markets. En Y. Bondi, A. Canziani y T. Kirat (Eds.), The Firm as an entity: implications for economics, accounting and the law (pp. 54-72). Routledge.

Villar, M. (2021, 4 de mayo). El futuro de la Inteligencia Artificial en el turismo. INVAT-TUR. https://bit.ly/3iimyKv

Weber, M. (1928). General economic history. (Frank H. Knigth, Trad.). The Economic Journal, 38(151), 462-465. (Obra original publicada en 1927). https://doi. org/10.2307/2224333 